TRANSACTIONS OF THE

AMERICAN MATHEMATICAL SOCIETY

Volume 355, Number 1, Pages 143-154

S 0002-9947(02)03133-1

Article electronically published on September 6, 2002

\title{
LEVI FOLIATIONS IN PSEUDOCONVEX BOUNDARIES AND VECTOR FIELDS THAT COMMUTE APPROXIMATELY WITH $\bar{\partial}$
}

\author{
EMIL J. STRAUBE AND MARCEL K. SUCHESTON
}

\begin{abstract}
Boas and Straube proved a general sufficient condition for global regularity of the $\bar{\partial}$-Neumann problem in terms of families of vector fields that commute approximately with $\bar{\partial}$. In this paper, we study the existence of these vector fields on a compact subset of the boundary whose interior is foliated by complex manifolds. This question turns out to be closely related to properties of interest from the point of view of foliation theory.
\end{abstract}

\section{INTRODUCTION}

In [7, Boas and Straube established a general sufficient condition for global regularity of the $\bar{\partial}$-Neumann problem in terms of (families of) vector fields that commute approximately with $\bar{\partial}$. They then showed that when the infinite type points lie in certain submanifolds, in particular in complex submanifolds, of the boundary, then there is an element in the first de Rahm cohomology of the submanifold that is the obstruction to the existence of the required vector fields: these vector fields exist if and only if this cohomology class is zero (7], Main Theorem and Remark 5). In the case of a complex submanifold of the boundary, this cohomology class has a geometric interpretation as a measure of the winding of the boundary around the submanifold ([3]).

In this paper, we study the situation where there is a Levi foliation in the boundary. More precisely, we assume that the set $K$ of infinite type points (which is compact) is the closure of its interior $\stackrel{\circ}{K}$ (in the boundary), and that all these points are Levi flat (the Levi form vanishes identically). By the Frobenius theorem, $\stackrel{\circ}{K}$ is foliated by complex manifolds of codimension 1; this foliation is usually referred to as the Levi foliation of $\stackrel{\circ}{K}$. We start in section 2 by briefly recalling from $[7$ what is needed concerning vector fields that commute approximately with $\bar{\partial}$. In section 3 , we assume that the domain $\Omega$ is in $\mathbb{C}^{2}$, and that the boundary of $K$ (relative to $b \Omega$ ) is a smooth surface with only isolated generic complex tangencies. Under suitable hypotheses, we show that the (families of) vector fields from [7] exist (Theorem 1). The cohomology class mentioned above, on each leaf of the Levi foliation, turns out to coincide with the infinitesimal holonomy of the leaf. Thus the question of existence of vector fields commuting approximately with $\bar{\partial}$ is closely

Received by the editors September 15, 2000.

2000 Mathematics Subject Classification. Primary 32W05, 32T99; Secondary 53C12.

Research supported in part by NSF grant DMS-9801539.

Marcel K. Sucheston died tragically on April 24, 2000. The surviving author dedicates this paper to his memory. 
related to foliation theoretic properties of the Levi foliation. We elaborate on this theme in section 4; in particular, Sullivan's theory of foliation currents ([17, [18, [10]) yields a homological necessary and sufficient condition for the existence of the vector fields (Theorem 3 ).

\section{Vector fieldS that COMmUte APPRoXimately With $\bar{\partial}$}

On a bounded pseudoconvex domain, the $\bar{\partial}$-Neumann operator $N_{q}(1 \leq q \leq n)$ is the inverse of the complex Laplacian $\bar{\partial} \bar{\partial}^{*}+\bar{\partial}^{*} \bar{\partial}$ acting on $(0, q)$-forms. For background on the $\bar{\partial}$-Neumann problem, we refer the reader to [12] and to the recent survey [8]. We say that the $\bar{\partial}$-Neumann problem is globally regular in Sobolev spaces on a domain $\Omega$ if the operators $N_{q}$ map the spaces $W_{0, q}^{s}(\Omega)$ of $(0, q)$-forms with coefficients in $W^{s}(\Omega)$ continuously to themselves for $s \geq 0,1 \leq q \leq n$. Here, $W^{s}(\Omega)$ denotes the usual $\mathcal{L}^{2}$-Sobolev space of order $s$.

In [7], Boas and Straube showed that a sufficient condition for global regularity is the existence of families of vector fields that have certain approximate commutation properties with $\bar{\partial}$. Let $\Omega$ be a smooth $\left(C^{\infty}\right)$ bounded pseudoconvex domain in $\mathbb{C}^{n}$ with defining function $\rho$. Suppose there is a constant $C>0$ such that for every $\varepsilon>0$ there exists a vector field $X_{\varepsilon}$ of type $(1,0)$ whose coefficients are smooth in a neighborhood $U_{\varepsilon}$ in $\mathbb{C}^{n}$ of the set of boundary points of $\Omega$ of infinite type (which is compact, [1]) and such that

$$
\left|\arg X_{\varepsilon} \rho\right|<\varepsilon \quad \text { and } \quad C^{-1}<\left|X_{\varepsilon} \rho\right|<C \text { on } U_{\varepsilon}
$$

and

$$
\left|\partial \rho\left(\left[X_{\varepsilon}, \partial / \partial \bar{z}_{j}\right]\right)\right|<\varepsilon \text { on } U_{\varepsilon}, \quad 1 \leq j \leq n .
$$

Condition (2) says that the normal $(1,0)$-component of the commutators $\left[X_{\varepsilon}, \partial / \partial \bar{z}_{j}\right]$ should have modulus less than $\varepsilon$, i.e., $X_{\varepsilon}$ commutes approximately with $\partial / \partial \bar{z}_{j}$, $1 \leq j \leq n$, modulo terms of type $(0,1)$ and terms that are complex tangential. These terms are benign (they can be absorbed) as far as Sobolev estimates for the $\bar{\partial}$-Neumann problem are concerned (see [6], p. 83, formulas (2) and (3) for a precise statement), and so we still say that the fields $X_{\varepsilon}$ commute approximately with $\bar{\partial}$, although only the normal $(1,0)$-component of the commutator is required to be small. We also note that in (2), it suffices to consider commutators with tangential fields of type $(0,1)$, as one can always suitably extend fields from the boundary of $\Omega$ to the inside (see [6], p. 86).

When computing the normal $(1,0)$-components of the commutators in (21), a certain 1-form appears naturally. Let $\eta:=i(\partial \rho-\bar{\partial} \rho)$ and $T:=-i\left(L_{n}-\bar{L}_{n}\right)$, where $L_{n}=\left(2 /|\nabla \rho|^{2}\right) \sum_{j=1}^{n} \frac{\partial \rho}{\partial \bar{z}_{j}} \frac{\partial}{\partial z_{j}} . L_{n}$ is the complex normal, normalized so that $\eta(T) \equiv 1$ on $b \Omega$. Let $\alpha:=-\mathcal{L}_{T} \eta$, minus the Lie derivative of $\eta$ in the direction of $T$ ([11], [7]). (Note that $\eta$ and $T$ as defined here differ by factors of $i$ and $-i$, respectively, from the notation in [11], [7], so that both $\eta$ and $T$ are real. This does not affect $\alpha$.$) If Y$ denotes a (local) section of $T^{0,1}(b \Omega)$, then

$$
\alpha(Y)=2 \partial \rho\left(\left[L_{n}, Y\right]\right)
$$

(see [11, p. 92, 7], section 2). Let $\bar{L}_{1}, \ldots, \bar{L}_{n-1}$ be local sections of $T^{0,1}(b \Omega)$ which span the complex tangent space to $b \Omega$ near a boundary point $P$. Near $P$, a vector field $X$ on $b \Omega$ of type $(1,0)$, with $X \rho \neq 0$ on $b \Omega$, can be written as 
$X=e^{h} L_{n}+\sum_{j=1}^{n-1} a_{j} L_{j}$ (the $e^{h} L_{n}$ term is actually global). We have (note that $\left.L_{n} \rho=1 / 2\right)$

$$
\begin{aligned}
\partial \rho\left(\left[X, \bar{L}_{k}\right]\right) & =e^{h}\left[\frac{1}{2} \bar{L}_{k} h+\partial \rho\left(\left[L_{n}, \bar{L}_{k}\right]\right)\right]+\sum_{j=1}^{n-1} a_{j} \partial \rho\left(\left[L_{j}, \bar{L}_{k}\right]\right) \\
& =\frac{1}{2} e^{h}\left[\bar{L}_{k} h+\alpha\left(\bar{L}_{k}\right)\right]+\sum_{j=1}^{n-1} a_{j} \partial \rho\left(\left[L_{j}, \bar{L}_{k}\right]\right) .
\end{aligned}
$$

In general, one can use both $h$ and the $a_{j}$ 's to adjust the commutators ([6], [7, [8], Remark 1 below). Assume now there is a Levi foliation in the boundary, as discussed in section 1 . We denote this foliation by $\mathcal{F}$. If $\bar{L}_{k}$ is tangent to the leaves of $\mathcal{F}$, then $\partial \rho\left(\left[L_{j}, \bar{L}_{k}\right]\right)=0,1 \leq j \leq n-1$ (because $\bar{L}_{k}$ is a null direction of the Levi form, which is positive semi-definite on the complex tangent space). So we only have the function $h$ to work with. If there exists a real function $h$, smooth in a neighborhood of the set $K$ of points of infinite type, such that

$$
d h / \text { leaf }=-\alpha / \text { leaf }
$$

for all leaves of the Levi foliation, then the field $X:=e^{h} L_{n}$ will have properties (1) and (2) above in a suitably small neighborhood $U_{\varepsilon}$ of $K$ (by continuity). We note that (55) requires of course that the restriction of $\alpha$ to any leaf of the Levi foliation is closed, but this was shown to be the case in [7] (see the lemma in section 2). Furthermore, the definition of $\alpha$ involves a choice of defining function. However, it is easy to see that $\alpha$ 's resulting from different defining functions differ by an exact form on the leaves of $\mathcal{F}$, so that whether or not (5) can be solved does not depend on the defining function. Indeed, if $\tilde{\rho}=e^{g} \rho$, then one computes that the restriction of $\alpha$ to leaves of $\mathcal{F}$ changes by $d g$.

The following property of $\alpha$ will be useful. In terms of $\eta$, the Frobenius condition reads $d \eta \wedge \eta=0$. Consequently, there exists a one-form $\beta$ such that $d \eta=\beta \wedge \eta$. In fact, $\alpha$ is such a form, that is $d \eta=\alpha \wedge \eta$ on $K$. For this, see for example the discussion in chapter 2 of [19], in particular Proposition 2.2.

Remark 1. At this point, we can easily consider the case of a Levi foliation of higher codimension. That is, assume that the rank of the Levi form is constant on $K$, but possibly greater than zero (which is the flat case). If that rank is $p$, then $\stackrel{\circ}{K}$ is foliated by complex manifolds of dimension $n-1-p$. If there is a function $h$ (smooth in a neighborhood of $K$ ) satisfying (5), then vector fields $X_{\varepsilon}$ satisfying (1) and (2) may be obtained using the complex tangential term $\sum_{j=1}^{n-1} a_{j} L_{j}$ to adjust the commutators when $L_{k}$ is tangential to $b \Omega$, but is not tangential to a leaf of the foliation (note that in this case, $\partial \rho\left(\left[L_{k}, \bar{L}_{k}\right]\right) \neq 0$, so that we do get a contribution in (4) from these terms). Details on how to obtain these fields locally, and on how to patch the local fields to fields defined in neighborhoods $U_{\varepsilon}$ of $K$, may be found in [6] (proof of the lemma) and [7, section 3.

\section{Domains IN $\mathbb{C}^{2}$}

We assume now that $\Omega$ is in $\mathbb{C}^{2}$. Suppose that $K$ is connected and has a smooth connected boundary; more precisely, $K$ is the closure, relative to $b \Omega$, of the smoothly bounded domain $\stackrel{\circ}{K}$ (as a subset of $b \Omega$ ). Denote by $\Gamma$ the boundary (relative to $b \Omega$ ) 
of $K$ (or $\stackrel{\circ}{K}$ ). Then $\Gamma$ is a smooth compact orientable surface embedded in $\mathbb{C}^{2}$. Recall that a generic complex tangency of $\Gamma$ is either elliptic or hyperbolic: nearby, $\Gamma$ can be written, in suitable coordinates, as $w=|z|^{2}+\lambda \operatorname{Re} z^{2}+O\left(|z|^{3}\right)$, with $0 \leq \lambda<1$ (the elliptic case) or $1<\lambda$ (the hyperbolic case). This goes back to Bishop's paper [5], as does the index formula $\# e-\# h=2-2 g$, where $\# e$ and $\# h$ denote the number of elliptic and hyperbolic tangencies, respectively, and $g$ is the genus of $\Gamma$. (Compare the introduction in [13] for information on various indices relevant for real surfaces inside complex surfaces.) In our situation, the following point of view is useful. The Levi foliation on $\stackrel{\circ}{K}$ (still denoted by $\mathcal{F}$ ) is induced by the 1-form $\eta$, and there is a corresponding 1-dimensional foliation, with singularities, on $\Gamma$, induced by restricting $\eta$ to $\Gamma$. The singularities (i.e., the points of $\Gamma$ where $\eta / \Gamma=0)$ are precisely the complex tangencies. Moreover, this foliation is orientable (since $\Gamma$ is), and so yields a vector field with singularities at the complex tangencies. The index is 1 at an elliptic point and -1 at a hyperbolic point. Thus the above index formula results in this case from the Poincaré-Hopf index theorem (compare also 4], section 3 for a discussion along these lines).

At a hyperbolic point of $\Gamma$, there are, locally, two leaves of $\mathcal{F}$ that meet (they can be constructed by flowing along suitable complex tangential curves through the point). We make the (global) assumption that these two local leaves are distinct globally.

For terminology from foliation theory, in particular for the notions of (germinal) holonomy and of infinitesimal holonomy, respectively, of a leaf, we refer the reader to 9 , 10].

Theorem 1. Let $\Omega$ be a smooth bounded pseudoconvex domain in $\mathbb{C}^{2}$. Suppose that the set $K$ of infinite type points of $b \Omega$ is smoothly bounded (in $b \Omega$ ) and that its boundary $\Gamma$ is connected and has only isolated generic complex tangencies. Assume that the two leaves meeting at a hyperbolic point of $\Gamma$ have no other hyperbolic points in their closure (in $K$ ). If each leaf of the Levi foliation is closed (in $\stackrel{\circ}{K}$ ) and has trivial infinitesimal holonomy, then the vector fields discussed in section 2 exist. Consequently, the $\bar{\partial}-$ Neumann problem on $\Omega$ is globally regular.

We now comment further on the assumptions.

Remark 2. Note again that it is understood that the two local leaves meeting at a hyperbolic point are distinct globally. The assumption that leaves have at most one hyperbolic point in their closure (relative to $K$ ) is also a kind of a genericity assumption; it simplifies the non-Hausdorff structure of the leaf space $\stackrel{\circ}{K} / \mathcal{F}$. It will be clear from the proof of Theorem 1 how to handle more general situations, provided certain "compatibility" conditions (arising from the fact that the local determination of $h$ near hyperbolic points propagates along leaves, by virtue of the relation (5) ) are met.

Remark 3. In order to solve $d h /$ leaf $=-\alpha /$ leaf, $\alpha$ must of course represent the trivial de Rham cohomology class on each leaf. However, the vanishing of this cohomology class is equivalent to the triviality of the infinitesimal holonomy of the leaf. In fact, the infinitesimal holonomy of a leaf $L$ can be viewed naturally as an element in the first de Rham cohomology of $L$, and then it is the class of $\alpha$ ([10, Example 2.3.15). In this context, compare also Corollary 2 in the appendix of [1]; the computations in section 2 above (see in particular (4)) show that if $e^{h} L_{n}$ is holomorphic on $L$, 
then $d h /{ }_{L}+\alpha / L=0$ (taking into account that both $h$ and $\alpha$ are real). It will be seen in section 4 that the existence of a function $h$ as above is equivalent to the existence of a (smooth) closed one-form that defines the Levi foliation, and thus, under the additional assumptions in Theorem 1 the holonomy itself of each leaf is trivial (see, e.g., [19, Theorem 3.29). For completeness, we point out that the condition of vanishing holonomy (hence vanishing infinitesimal holonomy) is trivially satisfied if the leaves are simply connected (that is, they are analytic discs, by the classification of simply connected Riemann surfaces).

Remark 4. An example where there is a leaf with nontrivial holonomy may be found in [3], p. 21. Consider

$$
S_{R}:=\left\{(z, w) \in \mathbb{C}^{2}|| z|\leq 1,1 \leq| w \mid \leq R, \operatorname{Re}\left(z e^{i \log |w|^{2}}\right)=0\right\} .
$$

This is a Levi flat hypersurface in $\mathbb{C}^{2}$. It is shown in $\left[3\right.$ how to "cap off" $S_{R}$ to obtain a pseudoconvex domain $\Omega \subseteq \mathbb{C}^{2}$ such that $S_{R} \subseteq b \Omega$ and $b \Omega \backslash S_{R}$ is strongly pseudoconvex. $\Omega$ is a worm domain, but with the critical annulus fattened. The leaves of the Levi foliation on $S_{R}$ are given by

$$
L(c):=\left\{z=i c w^{-2 i}|1<| w|<R,| z \mid<1\right\},
$$

where $c \in \mathbb{R}$. On $L(0)$, $\alpha$ does not represent the trivial cohomology class; its integral around a concentric circle inside $L(0)$ (which is an annulus) is nonzero. Also note that the leaves $L(c)$ for $c \neq 0$ are not closed (they spiral towards $L(0)$ ). Barrett's arguments carry over from the case of the "standard" worm domains (2]) to show that the conclusion of Theorem 1 fails for such $\Omega$.

Remark 5. Given the results of [6], it is natural to ask whether in the situation of Theorem 1 there is a defining function that is plurisubharmonic on the boundary. It is not hard to see that $e^{h} \rho$ ( $h$ as in section 2 ) gives a defining function that is plurisubharmonic at points of $K$ if $h$ is extended from the boundary with suitable normal derivative. While this implication does not hold in general ([7, Remark 3), in the case at hand it is a special case of results in [16].

To prove Theorem 1, it suffices, by the discussion in section 2, to find a function $h \in C^{\infty}(K)$ such that

$$
d h / \text { leaf }=-\alpha / \text { leaf. }
$$

We will construct $h$ on $\stackrel{\circ}{K}$ satisfying ([6) and, in addition, having all its derivatives bounded on $\stackrel{\circ}{K}$. Such a function will then extend to a function in $C^{\infty}(K)$ (since $\stackrel{\circ}{K}$ has smooth boundary). The construction proceeds in three stages: in saturated neighborhoods of the complex tangencies of $\Gamma$, in a saturated neighborhood of the closure of the remaining points, and, finally, patching to obtain a globally defined $h$. In the last step, the key is that we can use cutoff functions which are constant on the leaves (i.e., we only need to cut off in the direction transverse to the Levi foliation), so that (6) is not affected (this technique occurs in a related context already in [3]).

We start with a hyperbolic point $P$. There is a diffeomorphism of a neighborhood of $P$ (in $b \Omega$ ) onto a neighborhood of $O \in \mathbb{R}^{3}$ such that in these coordinates, $K$ is given locally by $x_{3} \leq x_{1}^{2}-x_{2}^{2}$ (by the Morse theorem). The complex tangent plane to $b \Omega$ at $P$ corresponds to the $\left(x_{1}, x_{2}\right)$-plane. Denote by $L_{1}$ the leaf of $\mathcal{F}$ that meets $O$ (i.e. $P$ ) from the side where $x_{1}<0$. (Near the origin, this leaf is obtained by 
flowing along complex tangential curves emanating from 0; alternatively, one starts at points on the (unique) complex tangential curve in the $\left(x_{1}, x_{3}\right)$-plane through 0 , and then flows along complex tangential directions transverse to it.) Then if $a>0$ is small enough, there is a unique $t_{0}$ such that $\left(-a, 0, t_{0}\right) \in L_{1}$. Then, for $t$ close enough to $t_{0}$, the curve $t \mapsto(-a, 0, t)$ is transversal to the leaves of $\mathcal{F}$, and it meets each leaf at most once. This last property is clear locally, but it also holds globally by [14, Proposition 3.1 (note that the assumption that the first Betti number of the underlying manifold, in our case $\stackrel{\circ}{K}$, is finite, is satisfied). On these leaves, we define $h$ (globally) by the requirements that

$$
h(-a, 0, t)=0
$$

and

$$
d h / \text { leaf }=-\alpha / \text { leaf } .
$$

We use here that $\alpha$ represents the trivial de Rham cohomology class on each leaf, or, equivalently, that each leaf has trivial infinitesimal holonomy (see Remark B above). Now consider the leaf $L_{2}$ which meets 0 from the side where $x_{1}>0$. There is a unique $t_{1}$ such that $\left(a, 0, t_{1}\right) \in L_{2}$ (again, we take $a>0$ small enough), and if $t$ is close enough to $t_{1}$, the curve $t \mapsto(a, 0, t)$ is transversal to $\mathcal{F}$ and meets each leaf at most once. If $t<t_{1},(a, 0, t)$ belongs to a leaf that passes through a point $\left(-a, 0, t^{\prime}\right)$ for some $t^{\prime}<t_{0}$. On these leaves, $h$ was defined above. Note that the function $h(a, 0, t)$ is smooth up to $t=t_{1}$ (from the side $t<t_{1}$ ). To see this, note that $h(a, 0, t)$ may be obtained by line integrals of $\alpha$ along integral curves of the vector field induced in the $\left(x_{1}, x_{3}\right)$-plane (near the origin) by intersecting with the complex tangent space. Both this vector field and $\alpha$ are smooth in a full neighborhood of the origin (in the $\left(x_{1}, x_{3}\right)$-plane). Denote by $g(t)$ a smooth continuation of $h(a, 0, t)$ across $t_{1}$. On the leaves where $t>t_{1}$, we now define $h$ (globally) by again requiring (8)), but replacing (77) by $h(a, 0, t)=g(t)$. (As above, we use that, by assumption, $\alpha$ is exact on the leaves of $\mathcal{F}$.) In order to do this, we need to know that for $t$ close enough to $t_{1}$ (and $t>t_{1}$ ), these leaves are distinct from the ones through points $\left(-a, 0, t^{\prime}\right)$, with $t^{\prime}>t_{0}$ and close to $t_{0}$. Since $L_{1}$ and $L_{2}$ have only one hyperbolic point, namely the origin, in their closure, and no elliptic point (no elliptic point is contained in the closure of a leaf by the regularity and uniqueness results in [15]), it follows that their closures intersect only at the origin, and they are smoothly bounded away from the origin (by an integral curve of the vector field induced on $\Gamma$ by $\eta / \Gamma)$. Consequently, away from the origin, there is transverse uniformity "up to the boundary" (see [9], Theorem 3, chapter III) for $L_{1}$ and $L_{2}$. This implies that if $t^{\prime}$ and $t$ are sufficiently close to $t_{0}$ and $t_{1}$, respectively, the above leaves are indeed distinct. Note that we have used here the hypothesis that $L_{1}$ and $L_{2}$ are distinct. Finally, observe that an argument similar to the one used above to show that $h(a, 0, t)$ is smooth up to $t=t_{1}$ shows that the $x_{3}$-derivatives of $h$ are bounded near the origin. Since complex tangential derivatives of $h$ are equal to components of $\alpha$, we get inductively that near the origin, all derivatives of $h$ are bounded, i.e., $h$ is smooth up to the boundary.

We have now extended $h$ into a saturated neighborhood of $P$ (this is a slight abuse of language, since $P$ is not in $\stackrel{\circ}{K}$ ). The extended function is $C^{\infty}$ on this saturated subset of $\stackrel{\circ}{K}$ (observe that the extension of $h$ along the leaves can be obtained via line integrals of $\alpha$, so that the smoothness of $\alpha$, together with the 
smoothness of $h$ near $P$, gives differentiability of $h$ also in directions transverse to the leaves; compare in this context the discussion of the holonomy maps in [9], chapter IV).

The function $h$ is constructed in a saturated neighborhood of the union of the hyperbolic leaves (leaves having a hyperbolic point in their closure) by repeating the above construction for every hyperbolic point. We use here the assumption that two leaves meeting at a hyperbolic point have positive distance from the remaining hyperbolic points. This means that if $L_{1}$ and $L_{2}$ meet at $P_{1}$, and $L_{3}$ and $L_{4}$ meet at $P_{2}$, then there are disjoint saturated neighborhoods of $L_{1} \cup L_{2}$ and $L_{3} \cup L_{4}$ (this argument is similar to the one used in the paragraph before the last, or see below, where we show that two leaves whose closures contain no hyperbolic points can be separated by saturated neighborhoods). Consequently, this construction gives a well-defined function $h$ that is $C^{\infty}$ in a saturated neighborhood of the union of the hyperbolic leaves.

A similar construction works near the elliptic complex tangencies. Near such a point, $\stackrel{\circ}{K}$ is foliated by a smooth one-parameter family of disks, and there is a (uniformly) transverse curve emanating from the elliptic point (see [15]). Prescribing $h$ to be zero on this curve and then solving $d h=\alpha$ on each leaf gives the required function $h$ in a saturated subset of $\stackrel{\circ}{K}$ near the elliptic points. Note that the disks close to an elliptic point do not contain other complex trangencies in their closure (by the regularity up-to-the-boundary results in [15]), so that we choose the saturated neighborhoods small enough to be pairwise disjoint and disjoint from a saturated neighborhood of the union of the hyperbolic leaves.

For the second step of the construction, we consider the leaf space $\stackrel{\circ}{K} / \mathcal{F}$ : points in the same leaf are identified, and the topology is the quotient topology. $\stackrel{\circ}{K} / \mathcal{F}$ is in general not Hausdorff (two hyperbolic leaves whose closure intersects in a hyperbolic point cannot be separated by saturated neighborhoods, and thus give rise to a pair of points in $\stackrel{\circ}{K} / \mathcal{F}$ which cannot be separated). In our situation, $\stackrel{\circ}{K} / \mathcal{F}$ carries a natural structure of a $C^{\infty}$ one-dimensional real (in general non-Hausdorff) oriented manifold ([14], section 3). We use here that the leaves of $\mathcal{F}$ are closed. Also, note that the first Betti number of $\stackrel{\circ}{K}$ is finite (this is assumed in all of section 3 in [14). The local charts are obtained as follows. Pick a point $P$ on a leaf $L$ and choose a (short) transversal $C^{\infty}$ curve through $P$ that meets each leaf of $\mathcal{F}$ at most once (14], Proposition 3.1). The projection of this curve gives a neighborhood $U$ of $L$ in $\stackrel{\circ}{K} / \mathcal{F}$, and we take a smooth parameter on the curve (for instance, arclength) to be a local coordinate in $U$. Then the coordinate transformations are given by holonomy maps and are thus $C^{\infty}([9]$, chapter IV). Because $\mathcal{F}$ is transversely oriented (by the vector field $T), \stackrel{\circ}{K} / \mathcal{F}$ is oriented. Note that the projection $\pi: \stackrel{\circ}{K} \rightarrow \stackrel{\circ}{K} / \mathcal{F}$ is a $C^{\infty}$ submersion.

Denote by $L_{1}, L_{2}, \ldots, L_{m}$ the hyperbolic leaves, and set $H:=L_{1} \cup L_{2} \cup \cdots \cup L_{m}$. Since the leaves are closed in $\stackrel{\circ}{K}, \stackrel{\circ}{K} \backslash H$ is open. Consider now the quotient $(\stackrel{\circ}{K} \backslash H) / \mathcal{F}$ (which is diffeomorphic to $\left.(\stackrel{\circ}{K} / \mathcal{F}) \backslash\left\{L_{1}, \ldots, L_{m}\right\}\right)$. We claim that it is Hausdorff. The argument is similar to one used above. First note that any leaf $L$ in $\stackrel{\circ}{K} \backslash H$ is smooth up to $\Gamma$ and intersects $\Gamma$ in an integral curve of the vector field induced on $\Gamma$ by $\eta / \Gamma$. The reason is that the closure $\bar{L}$ of $L$ in $K$ contains neither hyperbolic 
points (by the definition of $H$ ) nor elliptic points (no elliptic point is contained in the closure of a leaf of $\stackrel{\circ}{K}$, by the results of [15]). But near every other point $P$, $\mathcal{F}$ is smooth up to $\Gamma$ and there is a unique leaf that intersects $\Gamma$ (from one side) transversally in an integral curve through $P$ of the field induced by $\eta$. This implies that if $L_{1}$ and $L_{2}$ are two distinct leaves in $\stackrel{\circ}{K} \backslash H$, then they (equivalently, their closures) are a positive distance apart. For if not, their closures (in $K$ ) would have to intersect (in points of $\Gamma$ ). This is impossible by the preceding discussion. Moreover, for these leaves, there is again transverse uniformity (see 9], Theorem 3, chapter III) "up to the boundary". Consequently, they can be separated by saturated neighborhoods. This proves that $(\stackrel{\circ}{K} \backslash H) / \mathcal{F}$ is Hausdorff. With the differential structure induced as in the case of the full leaf space (equivalently, the restriction of the differential structure on $\stackrel{\circ}{K} / \mathcal{F}$ to $\left.\stackrel{\circ}{K} / \mathcal{F} \backslash\left\{L_{1}, \ldots, L_{m}\right\}\right),(\stackrel{\circ}{K} \backslash H) / \mathcal{F}$ is thus a bona fide real 1-dimensional manifold. By the classification of such manifolds, its connected components are diffeomorphic either to $\mathbb{R}$ or to $S^{1}$.

We now construct $h$ on the components of $\stackrel{\circ}{K} \backslash H$ (which correspond to components of $(\stackrel{\circ}{K} \backslash H) / \mathcal{F})$ as follows. Let $U$ be such a component. Then by the above, $\pi(U)$ is diffeomorphic to $\mathbb{R}$ or to $S^{1}$. Denote by $\gamma$ the one-form on $\pi(U)$ corresponding to $d x$ in the case of $\mathbb{R}$, and $d \theta$ in the case of $S^{1}$. Note that $\gamma$ is closed and nonvanishing. The form $\omega:=\pi^{*} \gamma$ is then a nonsingular ( $\pi$ is a submersion) one-form on $U$ that satisfies

$$
d \omega=d \pi^{*} \gamma=\pi^{*} d \gamma=0,
$$

and

$$
\omega / L=0,
$$

where $L$ is any leaf of $\mathcal{F} / U$. Thus $\omega$ is of the form $e^{g} \eta$ for some $C^{\infty}$-function $g$ (where $e^{g}=\omega(T)$ ). Therefore,

$$
0=d \omega=e^{g}(d g \wedge \eta+d \eta)=e^{g}(d g \wedge \eta+\alpha \wedge \eta) .
$$

Here we have used that on $K, d \eta=\alpha \wedge \eta$ (see the discussion at the end of section 2). Consequently,

$$
(d g+\alpha) \wedge \eta=0,
$$

which is equivalent to

$$
d g / L=-\alpha / L
$$

on each leaf of $\mathcal{F} / U$. (This computation will also show the equivalence of (i) and (ii) in Proposition 2 below.)

In the last step, we patch together the functions $h$ and $g$ constructed so far. By working in the leaf space (equivalently, on (short) integral curves of $T$ ), one constructs a smooth function $\psi$ which is constant on the leaves of $\mathcal{F}$, is identically one in a (saturated) neighborhood of $L_{1} \cup \cdots \cup L_{m}$, is supported in a small (saturated) neighborhood of this set, is identically one near elliptic points and is supported close to these points. Then the function $h_{1}:=\psi h+(1-\psi) g$ satisfies, for a leaf $L$ 
of $\mathcal{F}$,

$$
\begin{aligned}
d h_{1} / L=d(\psi h+(1-\psi) g / L & =(\psi d h+(1-\psi) d g) / L \\
& =\psi d h / L+(1-\psi) d g / L \\
& =\psi(-\alpha) /{ }_{L}+(1-\psi)(-\alpha) / L=(-\alpha) /{ }_{L} .
\end{aligned}
$$

That is, $h_{1}$ satisfies (6)).

It remains to check smoothness up to the boundary. As indicated at the beginning of the proof of Theorem [1, we will verify that derivatives of all orders are bounded. We have already noted that, by our construction, in local coordinates near a hyperbolic point of $\Gamma$, this is the case. The essence of the argument is the same near the other points of $\Gamma$. Let $P$ be a point of $\Gamma$ which is not a complex tangency. Near $P$, the Levi foliation is smooth up to $\Gamma$, the boundaries of the leaves being integral curves of the vector field on $\Gamma$ induced by $\eta / \Gamma$. Denote by $L_{p}$ the leaf "through" $P$. For $Q \in L_{p}$, sufficiently close to $P$, let $\sigma(t),-\varepsilon<t<\varepsilon$, be a short integral curve of $T$ through $Q$ (say $\sigma(0)=Q)$. For $z \in \stackrel{\circ}{K}$ near $P$, denote by $L_{z}$ the leaf of $\mathcal{F}$ through $z$, and by $t_{z}$ the unique $t$ such that $\sigma\left(t_{z}\right) \in L_{z}$. Writing $h_{1}(z)$ as $h_{1}\left(\sigma\left(t_{z}\right)\right)$ plus a line integral of $\alpha$ in $L_{z}$ shows, in view of the smoothness of $\alpha$ and of $\mathcal{F}$ up to $\Gamma$, that derivatives of all orders of $h_{1}$ are bounded near $P$. Analogous arguments work near an elliptpic point. This completes the proof of Theorem 1 .

Remark 6. Near an elliptic point, one can take advantage of the fact that conditions (1) and (2) need only be satisfied to within $\varepsilon$. Specifically, if $P$ is an elliptic point, define $h_{\varepsilon}$ near $P$ through $e^{h_{\varepsilon}} L_{n}=L_{n}(P)$. Then, close enough to $P$, $\left|\arg e^{h_{\varepsilon}} L_{n}(\rho)\right|<\varepsilon$, and $\partial \rho\left(\left[e^{h_{\varepsilon}} L_{n}, \partial / \partial \bar{z}_{j}\right]\right)=0$ (the latter holds since $e^{h_{\varepsilon}} L_{n}$ has holomorphic (namely constant) coefficients).

\section{More foliation theORY}

The existence of the function $h$ constructed above also entails some properties of interest from the point of view of foliation theory, both of the Levi foliation and of the flow generated by the transverse field $T$. This leads to a homological condition that is necessary and sufficient for the construction from section 3 , and thus for the vector fields from section 2 , with $\varepsilon=0$ on $K$, that uses Sullivan's theory of foliation currents $([17,18],[10])$. Although this does not seem to simplify the proof of, for example, Theorem [1 it is of independent interest, and we expect this point of view to be useful in the more general situation where the set $K$ of infinite type points is not the closure of a nicely foliated open subset of the boundary.

We keep the notation from section 2. For the moment, we let $M$ be a $C^{\infty}$-smooth (open) Levi flat hypersurface in $\mathbb{C}^{n} \cdot \mathcal{F}$ again denotes the Levi foliation on $M$ (in this case by complex manifolds of dimension $n-1$ ).

A one-form $\omega$ is said to define the foliation $\mathcal{F}$ if for each $P \in M$, the tangent space to the leaf through $P$ is equal to the kernel of $\omega$ at $P$. So the form $\eta$ defined in section 2 always defines $\mathcal{F}$.

A flow is said to be geodesible if there exists a Riemannian metric (on the underlying manifold) such that the orbits become geodesics (see, e.g., [19], chapter 6). We consider the flow generated by $T$. 
Proposition 2. The following are equivalent:

(i) there exists $h \in C^{\infty}(M)$ such that

$$
d h{ }_{L}+\alpha /{ }_{L}=0, \quad \forall L \in \mathcal{F}
$$

(ii) there exists a smooth $\left(C^{\infty}\right)$ closed one-form $\omega$ on $M$ that defines $\mathcal{F}$;

(iii) the flow generated by $T$ is geodesible by a metric that is obtained from the Euclidean metric on $M$ by scaling in the T-direction.

Note that (ii) and (iii) are manifestly independent of a choice of defining function, while the definition of $\alpha$ involves such a choice. However, we have pointed out already in section 2 that one can check by direct computation that (i) is actually independent of this choice.

Proof. As noted in section 3, the equivalence of (i) and (ii) follows from the computation (11)-(13) there. Namely, since $\omega$ defines $\mathcal{F}$, it is nonsingular; so $\omega=e^{h} \eta$ for some real-valued function $h$ (possibly after replacing $\omega$ by $-\omega$ ). Then

$$
\begin{aligned}
d \omega=d\left(e^{h} \eta\right) & =e^{h}(d h \wedge \eta+d \eta) \\
& =e^{h}(d h+\alpha) \wedge \eta .
\end{aligned}
$$

Therefore, $d \omega=0 \Leftrightarrow(d h+\alpha) \wedge \eta=0 \Leftrightarrow$ (i) holds. In the last equality in (15), we have again used that $d \eta=\alpha \wedge \eta$ (see section 2).

The equivalence of (ii) and (iii) is contained in Proposition 6.7 of [19, where various characterizations are given for a flow to be geodesible. But the arguments show that (ii) is equivalent to the flow of $T$ being geodesible by a metric as in (iii). This completes the proof of Proposition 2

Sullivan ([18]) characterizes geodesible flows by a homological condition. His arguments are easily modified to obtain a characterization of the conditions in Proposition 2 (actually, the versions on the compact set $K$, rather than on an open hypersurface, as in Proposition 2). We return to the analogue of the setup in section 3 for a domain in $\mathbb{C}^{n}$. Denote by $\mathcal{T}$ the one-dimensional foliation whose leaves are the orbits of $T$. (This is actually a foliation of all of $b \Omega$.) We briefly recall the notions we need from the theory of foliation currents; for details, see [17] or [10], chapter 10 . Denote by $\mathcal{D}_{1}(K)$ the space of one-forms that are $C^{\infty}$ on $K$, with the usual Fréchet space topology. Its strong dual $\mathcal{D}_{1}(K)^{\prime}$ comprises the one-currents on $K . \mathcal{D}_{1}(K)$ and $\mathcal{D}_{1}(K)^{\prime}$ are mutually dual (i.e., $\mathcal{D}_{1}(K)$ is reflexive). The closed convex cone $C_{\tau}$ of foliation currents for $\mathcal{T} / K$ is the closure, in $\mathcal{D}_{1}(K)^{\prime}$, of the set of all finite linear combinations with positive coefficients of currents given by pairing at $x$ with $T(x)$, for $x \in K$ (so-called Dirac currents). The crux of the matter is that $C_{\tau}$ has a compact base: $C_{\tau} \cap \eta^{-1}(0)=\{0\}$ and $C_{\tau} \cap \eta^{-1}(1)$ is compact (where $\eta$ is viewed as an element of $\left.D_{1}(K)^{\prime \prime}\right)$; this is analogous to Lemma 10.2.3 in [10. We say that a $p$-chain is tangential to $\mathcal{F}$ if it is a sum of $p$-chains, each of which is a $p$-chain in a leaf of $\mathcal{F}$.

Theorem 3. There exists $h \in \mathcal{D}(K)$ satisfying $d h /{ }_{L}+\alpha / L=0$ for each leaf $L$ of $\mathcal{F}$ if and only if no nontrivial foliation current for $\mathcal{T} / K$ can be arbitrarily well approximated in $\mathcal{D}_{1}(K)^{\prime}$ (i.e., uniformly on bounded subsets of $\mathcal{D}_{1}(K)$ ) by sums of the form (smooth 1-chain tangential to $\mathcal{F}$ ) plus (boundary of smooth 2 -chain in $\stackrel{\circ}{K}$ ). 
The proof of Theorem [3 is completely analogous to the proof of (iii) in the theorem in [18. Namely, the second statement in Theorem 3 means that the closed linear subspace of $\mathcal{D}_{1}(K)^{\prime}$ generated by boundaries of smooth 2 -chains in $\stackrel{\circ}{K}$ and smooth 1-chains tangential to $\mathcal{F}$ intersects the cone $C_{\tau}$ only at $0 \in \mathcal{D}_{1}(K)^{\prime}$. Since $C_{\tau}$ has a compact base (see above), a suitable version of the Hahn-Banach theorem (see, e.g., 10], Theorem 10.2.5 and the remark immediately following it) yields an element $\omega \in \mathcal{D}_{1}(K)^{\prime \prime}=\mathcal{D}_{1}(K)$ that vanishes on the boundaries of smooth 2-chains and on smooth 1-chains tangential to $\mathcal{F}$, while it is strictly positive on $C_{\tau} \backslash\{0\}$. These properties imply, respectively, that $\omega$ is closed, vanishes at all points on vectors tangent to the leaf of $\mathcal{F}$ through the point, and is strictly positive on $T$ at all points of $K$. Consequently, $\omega=e^{h} \eta$, with $h \in \mathcal{D}(K)$, and $h$ has the required property, see (the proof of) Proposition 2,

On the other hand, if there exists $h$ as in the theorem, then $\omega=e^{h} \eta$ is closed (again by the proof of Proposition 2), and, if $\varphi \in C_{\tau}$, then $\varphi(\omega)>0$, so that $\varphi$ cannot be approximated as indicated in the theorem. This concludes the proof of Theorem 3

Remark 7. As an illustration, consider the following. We know from section 3 that if there exists a function $h$ as in Theorem 3 then the Levi foliation must be without holonomy. It is easily seen directly that the homological condition in Theorem 3 implies that the holonomy of $\mathcal{F}$ is trivial. Consider a point $x_{0}$ in a leaf $L$ and a simple loop $\sigma$ in $L$, starting and ending at $x_{0}$. Lift $\sigma$ to a nearby leaf as in the construction of the holonomy maps. This yields a path $\tilde{\sigma}$. If $\tilde{\sigma}$ is not a loop, we have a two-dimensional surface whose boundary consists of $\sigma$ plus $\tilde{\sigma}$ (both of which are tangential to the Levi foliation) plus a piece of an integral curve of $T$ (i.e., a nontrivial foliation current for $\mathcal{T}$ ). This is impossible by the homological condition. Since $\sigma$ was arbitrary, $L$ has trivial holonomy.

\section{REFERENCES}

[1] David Barrett: Complex analytic realization of Reeb's foliation of $S^{3}$, Math. Z. 203 (1990), 355-361. MR 91f:32018

[2] David Barrett: Behavior of the Bergman projection on the Diederich-Fornaess worm, Acta Math. 168, 1-2 (1992), 1-10. MR 93c:32033

[3] Eric Bedford and John Erik Fornaess: Domains with pseudoconvex neighborhood systems, Invent. Math. 47 (1978), 1-27. MR 58:17215

[4] Eric Bedford and Bernard Gaveau: Envelopes of holomorphy of certain 2-spheres in $\mathbb{C}^{2}$, American J. of Math. 105 (1983), 975-1009. MR 84k:32016

[5] Errett Bishop: Differentiable manifolds in complex Euclidean space, Duke Math. J. 32 (1965), 1-21. MR 34:369

[6] Harold P. Boas and Emil J. Straube: Sobolev estimates for the $\bar{\partial}$-Neumann operator on domains in $\mathbb{C}^{n}$ admitting a defining function that is plurisubharmonic on the boundary, Math. Z. 206 (1991), 81-88. MR 92b:32027

[7] Harold P. Boas and Emil J. Straube: De Rham cohomology of manifolds containing the points of infinite type, and Sobolev estimates for the $\bar{\partial}$-Neumann problem, J. Geometric Analysis 3, Nr. 3 (1993), 225-235. MR 94g:32019

[8] Harold P. Boas and Emil J. Straube: Global regularity of the $\bar{\partial}$-Neumann problem: a survey of the $\mathcal{L}^{2}$-Sobolev theory, Several Complex Variables, M. Schneider and Y.-T. Siu editors, Mathematical Sciences Research Institute Publications 37, 79-111, Cambridge Univ. Press, 1999.

[9] César Camacho and Alcides Lins Neto: Geometric Theory of Foliations, Birkhäuser, 1985. MR 87a:57029

[10] Alberto Candel and Lawrence Conlon: Foliations I, Graduate Studies in Mathematics, vol. 23, American Math. Society, 2000. MR 2002f:57058 
[11] John P. D'Angelo: Several Complex Variables and the Geometry of Real Hypersurfaces, CRC Press, 1993. MR 94i:32022

[12] G. B. Folland and J. J. Kohn: The Neumann Problem for the Cauchy-Riemann Complex, Annals of Math. Studies 75, Princeton Univ. Press, 1972. MR 57:1573

[13] Franc Forstnerič: Complex tangents of real surfaces in complex surfaces, Duke Math. J. 67 (1992), 353-376. MR 93g:32025

[14] André Häfliger: Variétés feuilletées, Ann. Scuola Norm. Sup. Pisa 16 (1962), 367-397. MR 32:6487

[15] Carlos E. Kenig and Sidney M. Webster: The local hull of holomorphy of a surface in the space of two complex variables, Invent. Math. 67 (1982), 1-21. MR 84c:32014

[16] Emil J. Straube and Marcel K. Sucheston: Plurisubharmonic defining functions, good vector fields, and exactness of a certain one form, Monatsh. Math. 136 (2002), 249-258.

[17] Dennis Sullivan: Cycles for the dynamical study of foliated manifolds and complex manifolds, Invent. Math. 36 (1976), 225-255. MR 55:6440

[18] Dennis Sullivan: A foliation of geodesics is characterized by having no "tangent homologies", J. Pure and Applied Algebra 13 (1978), 101-104. MR 80c:57019

[19] Philippe Tondeur: Geometry of Foliations, Birkhäuser, 1997. MR 98d:58037

Department of Mathematics, Texas A\&M University, College Station, Texas 778433368

E-mail address: straube@math.tamu.edu 